\title{
Visualizations of personal social networks on Facebook and community structure: an exploratory study
}

\author{
Christina Gkini ${ }^{1}$ \\ Alexios Brailas ${ }^{2}$
}

Published online: 30 J une 2015

\begin{abstract}
We studied the community structure pattern in the visualizations of ten personal social networks on Facebook at a single point in time. It seems to be a strong tendency towards community formation in online personal, social networks: somebody's friends are usually also friends between them, forming subgroups of more densely connected nodes. Research on community structure in social networks usually focuses on the networks' statistical properties. There is a need for qualitative studies bridging the gap between network topologies and their sociological implications. To this direction, visual representations of personal networks in social media could be a valuable source of empirical data for qualitative interpretation. Most of the personal social networks' visualizations in the present study are very highly clustered with densely-knit overlapping subgroups of friends and interconnected between them through wide bridges. This network topology pattern seems to be quite efficient, allowing for a fast spread and diffusion of information across the whole social network.

Keywords: social network analysis, social network visualization, community structure, Gephi, friendships network, Facebook
\end{abstract}

Acknowledgement: This is an original paper that presents personal views of the authors.

\section{Introduction}

Networks today are everywhere. The brain of all mammals is a vast network of neurons (nodes) connected through synapses (edges). The difference today, in comparison to the past, is that we are more sensitive to observe the ever present networks; now instead of looking at standalone entities, we focus more and more on their relation, on the connections between them: "Network thinking means focusing on relationships between entities rather on the entities themselves" (Mitchell, 2009. p. 233). The paradigm shift from the study of the isolated parts towards the study of the interconnected whole is prominent in many disciplines. Michael Bakhtin focused on the relation-between two entities quite before network thinking conquered scientific foreground: "all meaning is relative in the sense that

\footnotetext{
${ }^{1}$ Undergraduate student, Department of Psychology, Panteion University of Social and Political Sciences. Email: christina_gini@hotmail.com

${ }^{2}$ Researcher (PhD) contributing research to the Virtual Reality, Internet Research and eLearning Laboratory at the Department of Psychology, Panteion University of Social and Political Sciences. Email: abrailas@panteion.gr
} 
it comes about only as a result of the relation between two bodies occupying simultaneous but different space" (Holquist, 2002, p. 21). In this view, the self exists only through its relation to another self. In complexity theory, networks are complex entities with emergent properties so that the whole is quite different from the sum of the parts (Feldman, 2012). In literature, Italo Calvino, a famous writer, highlights, in a very vivid way, the importance of the relations for establishing human communities, when referring to the invisible city of Ersilia: "In Ersilia, to establish the relationships that sustain the city's life, the inhabitants stretch strings from the corners of the houses, white or black or gray or black-andwhite according to whether they mark a relationship of blood, of trade, authority, agency" (Calvino, 1974, p. 76).

\section{Background}

Personal social networks are usually characterized by community structure: somebody's friends are usually also friends between them, forming subgroups of more densely connected nodes on their network visual representation. This form of largely separate, tightly knit communities in networks is also termed clustering of the nodes (Mitchell, 2009). Community structure is an important aspect of personal social networks; communities of friends form the basis to understand the contexts of somebody's social life (Watts, 2004). Different clusters of friends are associated with different contexts in a person's life, for example with different interests, activities or different periods of life (Mitchell, 2009). According to Duncan Watts (2004): "By belonging to certain groups and playing certain roles, individuals acquire characteristics that make them more or less likely to interact with one another. Social identity, in other words, drives the creation of social networks" ( $p$. 116).

People and their social networks are always in a co-evolving transformative reciprocal relation. It works both ways: "People may know who they know because of what they do, but people also try new things because of who they know. Your friends invite you to parties or drag you along on their favorite activities" (Watts, 2004 , p. 128). An individual, in order to remain fit in a complex living landscape, must adapt to the adaptations of the network of all other agents (Kauffman, 1996). I shape my personal network, the very moment I am shaped by my personal network: "Many networks are the product of dynamical processes that add or remove vertices or edges. For instance, a social network of friendships changes as individuals make and break ties with others" (Newman et al, 2006, p. 7). Therefore, there is a relation of reciprocal determination between the form of people's friendship network and the way they connect to new friends: "The ties people make affect the form of the network, and the form of the network affects the ties people make. Social network structure therefore evolves in a historically dependent manner, in which the role of the participants and the patterns of behavior they follow cannot be ignored" (Newman et al, 2006, p. 7). A critical question to ask is: what story somebody's friendships network tell us about her personality? By studying the structure of a person's social network, we witness simultaneously the network's history as well its dynamics.

Previous research on networks' community structure has focused extensively on the statistical properties of the identified community structures in social networks and web groups. However, there is a need for qualitative studies bridging the gap between network topologies and behavioral or sociological 
implications. To this direction, visual representations of network data depicting their community structure and the pattern of interconnection among clusters could be a quite valuable source of empirical data for qualitative interpretation (Ferrara, 2012b).

\section{Method}

We study the personal social networks on Facebook of ten (10) participants in a senior undergraduate research seminar offered by the Psychology Department of Panteion University of Social and Political Sciences, in Athens, Greece, during the winter semester of the academic year 2014-15. The participants were female students, aged between 19 to 25 years old. Personal friendships network data were extracted from the participants' Facebook accounts, with their consent and cooperation, by using NetVizz application (Rieder, 2013). Network data were anonymized prior to any further analysis.

Following network data collection, the corresponding network graphs were analyzed using Gephi network analysis software (Bastian, Heymann, \& J acomy, 2009). The basic networks' characteristics (presented in ascending order of the number of friends/nodes) are shown in Table 1 . The first network analysis technique applied to each personal social network in Gephi was an outline algorithm, specifically ForceAtlas2 (J acomy, Heymann, Venturini, \& Bastian, 2012). This algorithm belongs to a class of imaging algorithms that operate through attraction-repulsion forces. The core principle of this algorithm is that the more densely interconnected nodes are attracted to each other while lesser interconnected ones are repelled (J acomy et al, 2012). By applying this outline algorithm, the nodes of each graph were eventually grouped together in community clusters based on the interconnections between them in the overall graph structure. By applying the ForceAtlas2 outline algorithm, a clear visual community structure appeared in every graph in our study. Subsequently, we applied a more specialized community detection algorithm on each graph.

Table 1: Personal social network of 60 nodes.

\begin{tabular}{|c|c|c|c|c|}
\hline $\begin{array}{c}\text { Nodes } \\
\text { (friends) }\end{array}$ & Edges & $\begin{array}{c}\text { Avg. } \\
\text { Degree }\end{array}$ & $\begin{array}{c}\text { Network } \\
\text { Diameter }\end{array}$ & $\begin{array}{c}\text { Avg. Path } \\
\text { Length }\end{array}$ \\
\hline 60 & 164 & 5.467 & 4 & 2.103 \\
\hline 108 & 191 & 3.537 & 7 & 2.95 \\
\hline 186 & 766 & 8.237 & 8 & 3.311 \\
\hline 220 & 1475 & 13.409 & 6 & 2.73 \\
\hline 377 & 5259 & 27.899 & 5 & 2.293 \\
\hline 452 & 3908 & 17.292 & 10 & 3.168 \\
\hline 507 & 2872 & 1.329 & 9 & 3.664 \\
\hline 698 & 6474 & 18.55 & 9 & 3.456 \\
\hline 940 & 10943 & 23.283 & 8 & 3.057 \\
\hline 1011 & 16308 & 32.261 & 8 & 3.014 \\
\hline
\end{tabular}

In social network analysis, community detection is the process of identifying sub-groups of people more densely interconnected in comparison to out-members 
(Blondel, Guillaume, Lambiotte, \& Lefebvre, 2008). The community detection method used in Gephi is based on an algorithm first proposed by Newman and Girvan, with minor modifications to improve computational performance (Blondel et al, 2008). The sensitivity of the Gephi community detection algorithm can be adjusted using a resolution coefficient (Lambiotte, Delvenne, \& Barahona, 2009). Increasing this resolution coefficient leads to detecting fewer and larger communities, while decreasing it leads to detecting more and smaller clusters. The detected communities in Gephi are visualized by a different color. The resolution coefficient was adjusted to 1.0 (default value) for the purposes of the exploratory analysis of our study. For each application of the algorithm a modularity parameter was computed (Blondel et al, 2008) and then used as a quality measure for the specific network partition in communities of friends. Values for the modularity over 0.4 are considered satisfactory and meaningful (Blondel et al, 2008). In every graph in our study, modularity parameter was within the accepted range of values.

\section{Network Visualizations - Results}

The personal social networks' visualizations of the participants in our study are presented in this section (Figures 1 to 10) in the ascending order of the number of nodes (Facebook friends) of each network, in order to reveal any possible pattern of community structure that is related to networks' sizes or that is persistent through all the graphs, regardless to their sizes. Different colors on the graphs' nodes denote different communities.

Figure 1: Personal social network of 60 nodes.

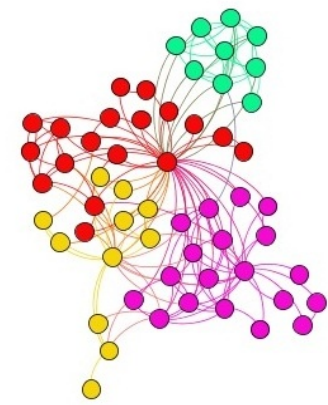

Figure 2: Personal social network of 108 nodes.

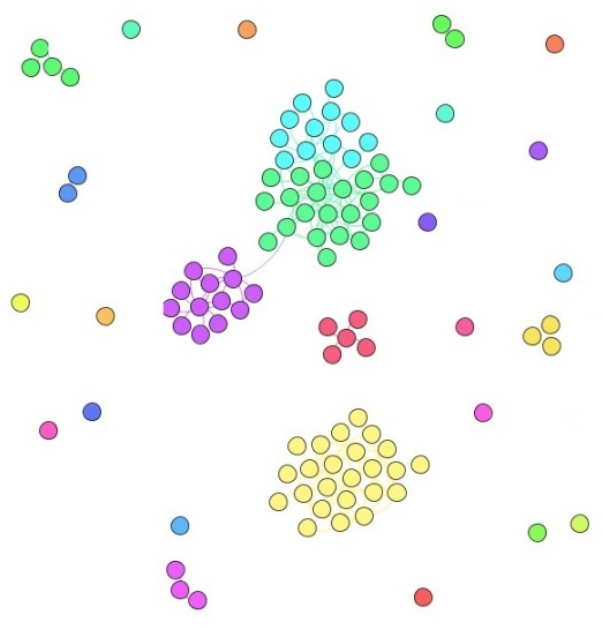


Figure 3: Personal social network of 186 nodes.

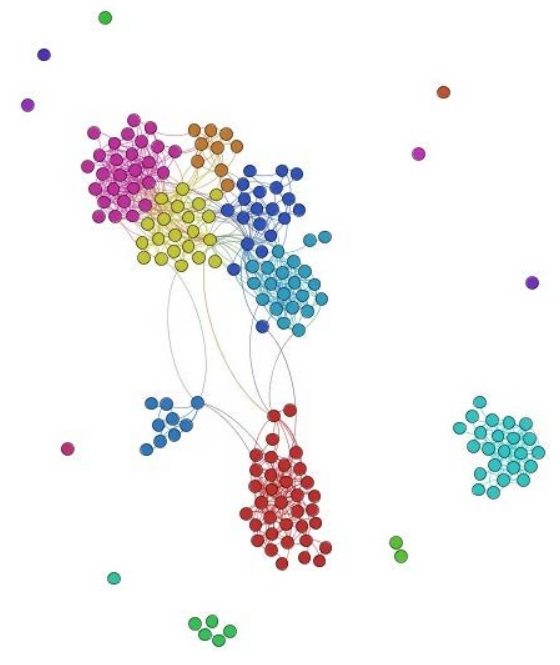

Figure 4: Personal social network of 220 nodes.

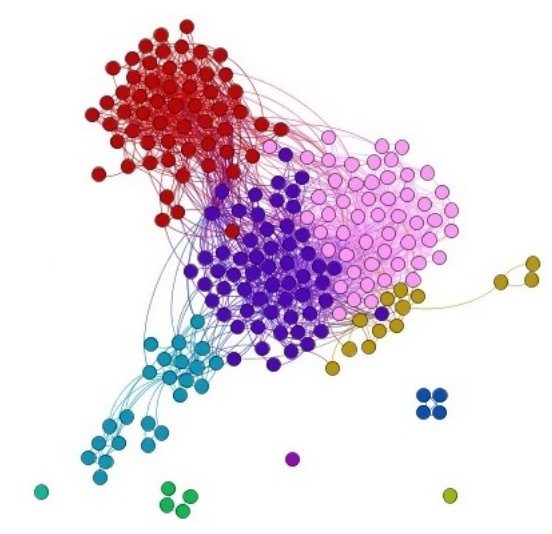

Figure 5: Personal social network of 377 nodes.

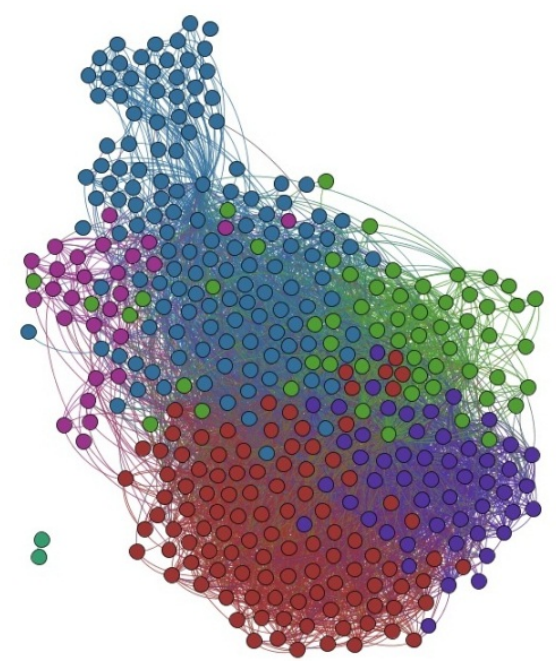


Figure 6: Personal social network of 452 nodes.

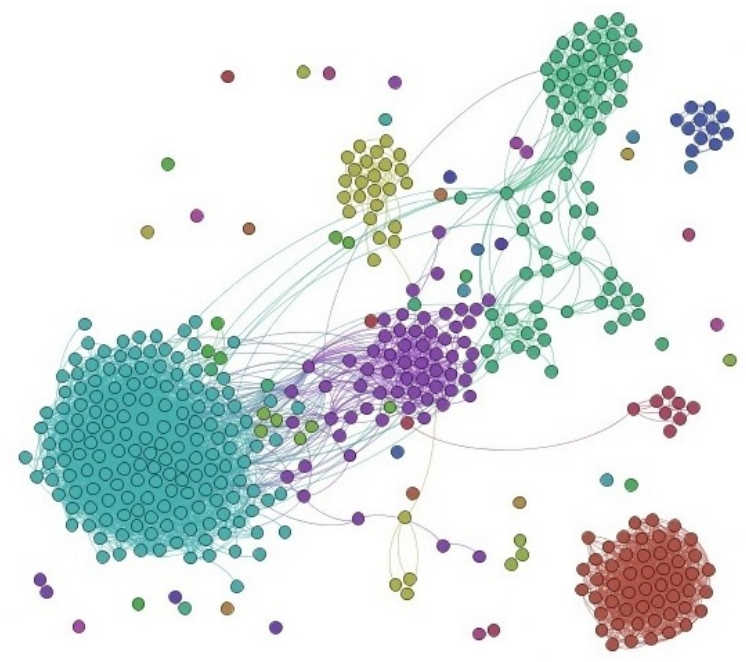

Figure 7: Personal social network of 507 nodes.

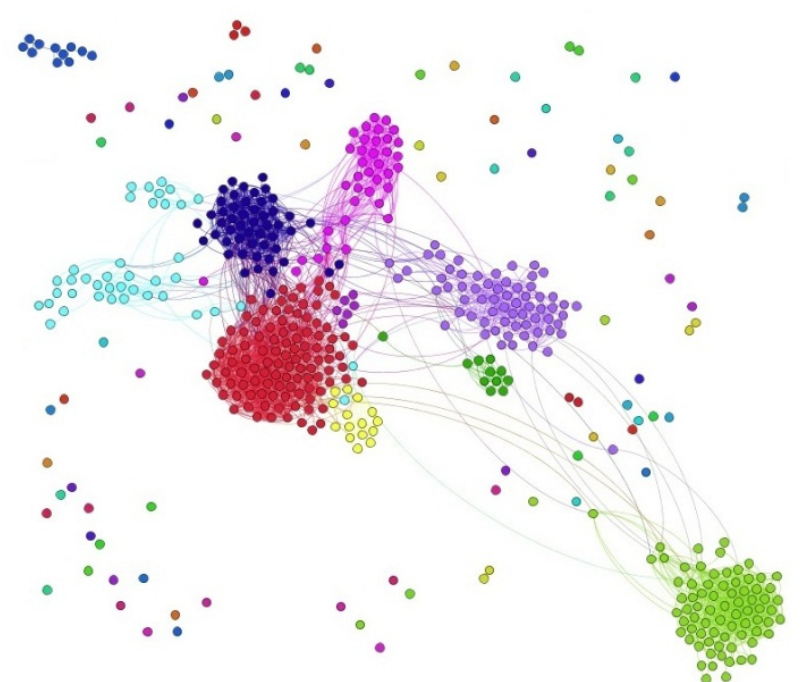

Figure 8: Personal social network of 698 nodes.

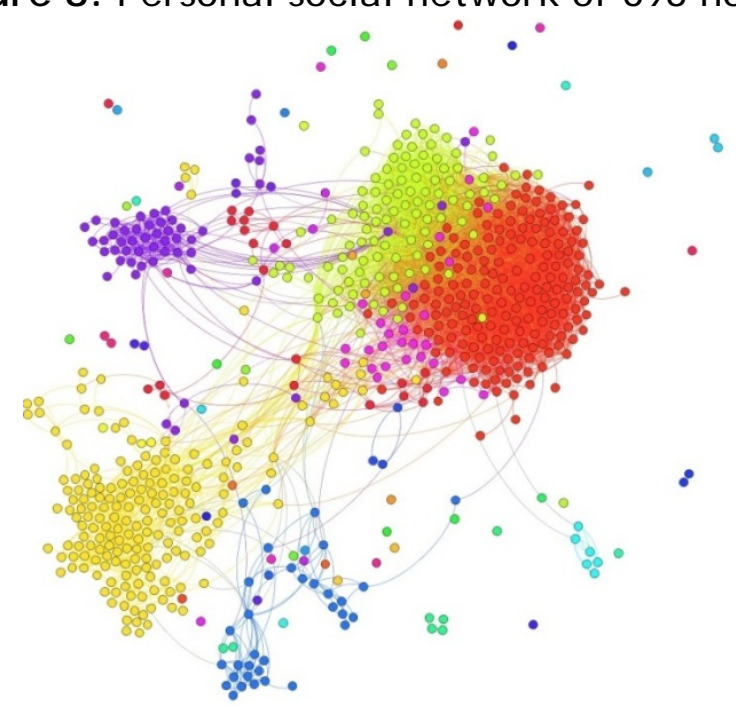


Figure 9: Personal social network of 940 nodes.

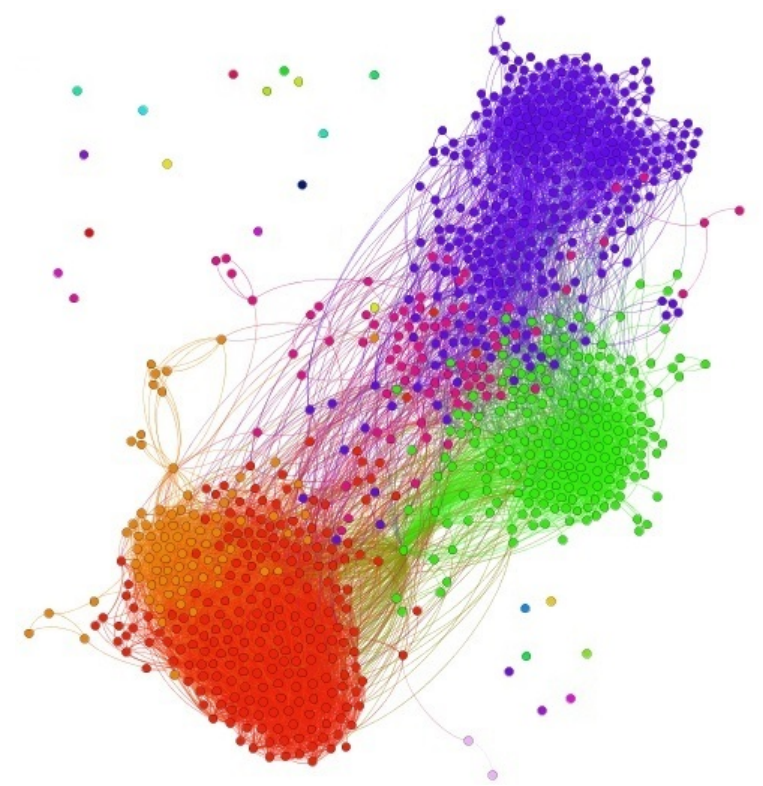

Figure 10: Personal social network of 1011 nodes.

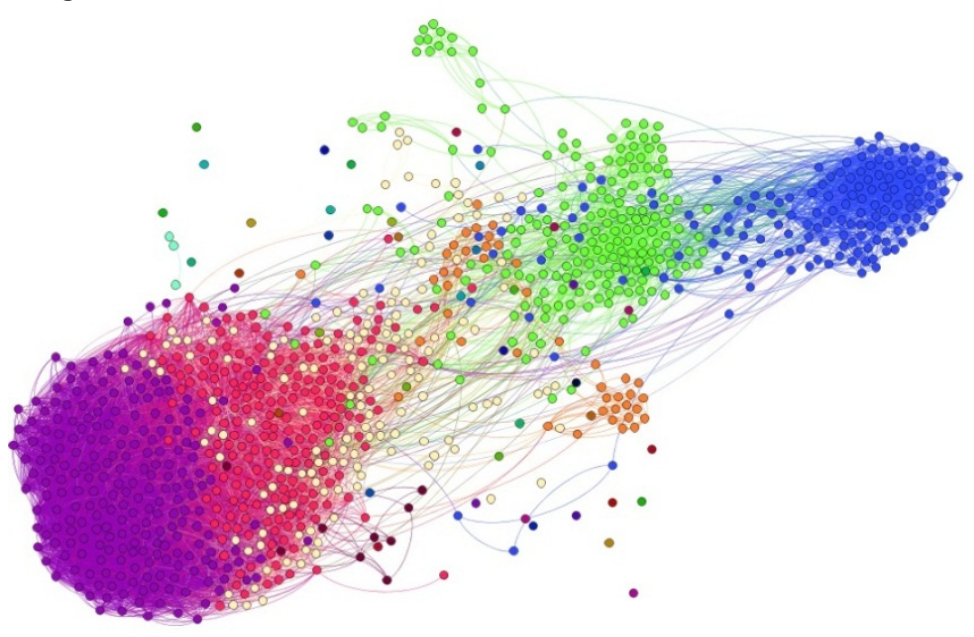

\section{Discussion}

It seems to be a strong tendency towards the formation of communities in online personal social networks. Most of the personal social networks' visualizations in our study are very highly clustered with densely-knit subgroups of friends (community structure). These observed subgroups are also interconnected between them through wide bridges (partially overlapping communities). Community structure and inter-community bridges are more evident as the number of nodes grows. This network topology of densely-knit subgroups of nodes interconnected through wide bridges, observed in most of the personal networks in our study, resembles strongly with the visualization of world flight routes based on openflights. org data, shown on Figure 11. World flight routes functions to support efficient air transportations at a global scale. 
Figure 11: World flight routes ${ }^{3}$.

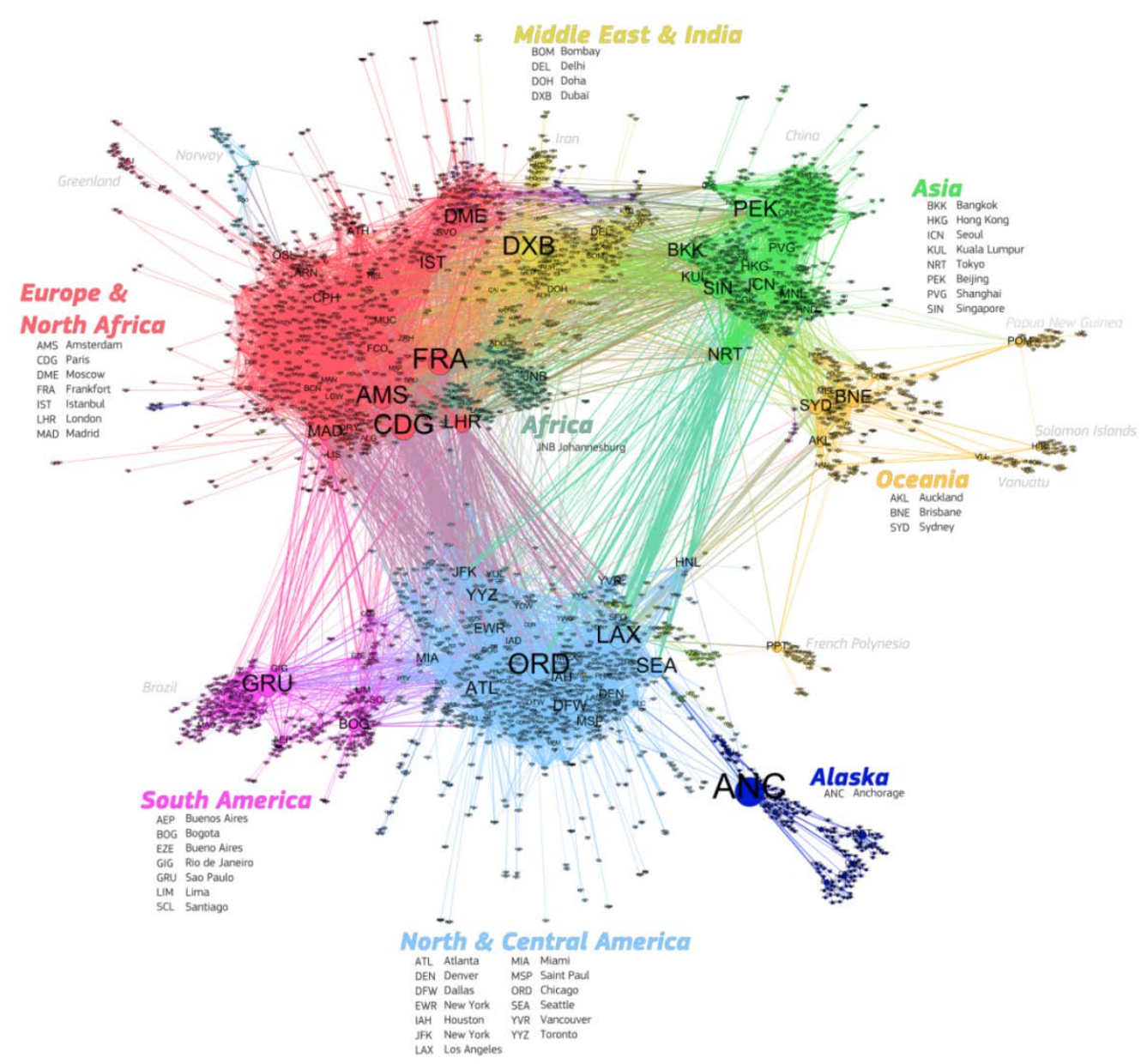

This network topology pattern, with discrete but bridged communities, appearing in many different contexts, seems to weave the necessary social fabric in order to efficiently support the dissemination of complex ideas, shared norms and social practices (Centola, 2015). People know each other because of the activities in which they participate (Watts, 2004), resulting in the formation of discrete communities of friends. These highly interconnected subgroups, which seem to indicate the presence of the small world phenomenon (Milgram, 1967; Watts \& Strogatz, 1998) that is usually observed in online social networks (Ferrara, 2012a), allow for a much faster spread and diffusion of information across the whole social network (Nguyen, Senac, \& Diaz, 2012). This diffusion of information inside the community might modify the individual's interests and activities and therefore reshape once again her own personal social network: "all the activities we pursue that lead us to meet and interact with each other are contexts. So the set of contexts in which each of us participates is an extremely important determinant of the network structure that we subsequently create" (Watts, 2004, p. 115).

The sample in our exploratory study (ten friendships' graphs) is quite small and further research is needed to investigate the persistence of the observed network topology through different cultural settings. Longitudinal studies are

\footnotetext{
${ }^{3}$ Image from Matthieu Totet based on data from http://openflights.org/data.html and analyzed with Gephi. Image under license CC BY SA, retrieved from http://matthieu-totet.fr/world_flight_routes.png
} 
required to investigate on the evolution of somebody's community network pattern through her different personal developmental phases (childhood, adolescence or adulthood). An interesting research question for a following up study is whether there is a relation between the network topology of somebody's friendships graph and the way that she connects and socializes with other persons, and whether it works the other way: is it possible after the network topology reaching a critical threshold pattern, to fire a change in the way a person socializes?

\section{References}

Bastian, M., Heymann, S., \& J acomy, M. (2009). Gephi: An open source software for exploring and manipulating networks. Presented at the International AAAI Conference on Weblogs and Social Media. Retrieved from http:/ / www. aaai. org/ ocs/ index. php/ ICWSM/ 09/ paper/ view/ 154

Blondel, V. D., Guillaume, J. L., Lambiotte, R., \& Lefebvre, E. (2008). Fast unfolding of communities in large networks. J ournal of Statistical Mechanics: Theory and Experiment, 10, 10008 (12 pp).

Calvino, I. (1974). Invisible Cities. USA: Harcourt Brace \& Company.

Centola, D. (2015). The Social Origins of Networks and Diffusion. American J ournal of Sociology, 120(5), 1295-1338. http:/ / doi.org/ 10.1086/681275

Feldman, D. P. (2012). Chaos and Fractals: An Elementary Introduction. UK: Oxford University Press.

Ferrara, E. (2012a). A large-scale community structure analysis in Facebook. EPJ Data Science, 1(1), 1-30. doi:10.1140/ epjds9

Ferrara, E. (2012b). Community structure discovery in Facebook. Int. J . of Social Network Mining, 2012 Vol. 1, No. 1, pp.67-90. 10.1504/ IJ SNM. 2012.045106

Holquist, M. (2002). Dialogism: Bakhtin and His World. New York: Routledge.

J acomy, M., Heymann, S., Venturini, T., \& Bastian, M. (2012). ForceAtlas2, a continuous graph layout algorithm for handy network visualization. Retrieved from http:// www. medialab. sciencespo.fr/publications/J acomy_Heymann_Venturini-Force_Atlas2.pdf

Kauffman, S. (1996). At Home in the Universe: The Search for the Laws of SelfOrganization and Complexity. USA: Oxford University Press.

Lambiotte, R., Delvenne, J. C., \& Barahona, M. (2009). Laplacian dynamics and multiscale modular structure in networks. Arxiv preprint arXiv:0812.1770. Retrieved from http:// arxiv.org/ pdf/ 0812.1770v3.pdf

Milgram, S. (1967). The small world problem. Psychology today, 2(1), 60-67. Mitchell, M. (2009). Complexity: A Guided Tour. USA: Oxford University Press. Newman, M., Barabási, A. L., \& Watts, D. J . (2006). The Structure and Dynamics of Networks. USA: Princeton University Press.

Nguyen, A. D., Senac, P., \& Diaz, M. (2012). Understanding and modeling the small-world phenomenon in dynamic networks. In Proceedings of the 15th ACM international conference on Modeling, analysis and simulation of wireless and mobile systems (pp. 377-384). Paphos, Cyprus: ACM.

Rieder, B. (2013). Studying Facebook via Data Extraction: The Netvizz Application. In Proceedings of the 5th Annual ACM Web Science Conference (pp. 346-355). Paris, France: ACM. http:/ / doi.org/ 10.1145/ 2464464. 2464475

Watts, D. (2004). Six Degrees: The New Science of Networks. UK: Random House.

Watts, D. J ., \& Strogatz, S. H. (1998). Collective dynamics of 'small-world' networks. Nature, 393(6684), 440-442. doi:10.1038/30918 


\section{Notes on Contributors}

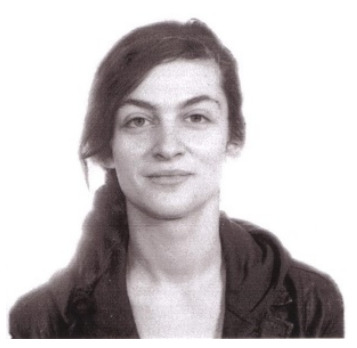

Christina Gkini is an undergraduate student in the Psychology Department at Panteion University of Social and Political Sciences. Her research interests are in Sociology of Body and Embodiment, Prison Sociology and Sociology of Information Technology.

Email: christina gini@hotmail.com

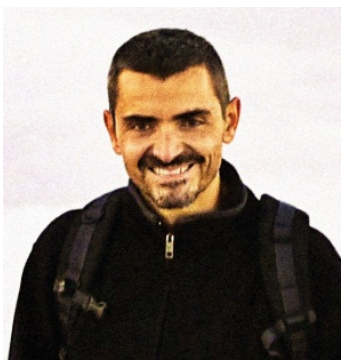

Alexios V. Brailas (PhD) is a researcher in education contributing research to the Virtual Reality, Internet Research and e-Learning Laboratory at the Department of Psychology, Panteion University of Social and Political Sciences (http:// vrlab.panteion.gr). He works in Athens, Greece, as an educator and as a psychologist. Through his research, he tries to bring insights from complexity theory and practices from group psychotherapy to formal and informal teaching. Among his Greek books are Complex Systems and Chaos: Psychosocial and pedagogical approaches (2015) and Learning at the Internet Chronotope: Communities, digital culture, Wikipedia \& MOOCs (2015).

E-mail: abrailas@yahoo.com. Web site: http://www.abrailas.com.

Correspondence regarding this article can be addressed to: Christina Gkini, E-mail: christina gini@hotmail.com 